\title{
Analysis of Clinical Characteristics of Cognitive Impairment in Patients with Acute Hemorrhagic Stroke
}

\author{
J. ZHOU, Z. Y. WANG ${ }^{1}$, H. B. CHEN, R. HU, A. H. GUO, Z. Li, H. M. HUANG, Y. YANG, J. M. YU, J. X. HU AND X. P. PAN \\ Department of Neurology, Guangzhou First People's Hospital, School of Medicine, South China University of Technology, No. \\ 602 Renmin Bei road, Guangzhou, Guangdong 510180, ${ }^{1}$ Department of Neurology, Dongguan MarinaBay Central Hospital, \\ No. 154 Humen Avenue, Dongguan, Guangdong 523900, China
}

\section{Zhou et al.: Analysis of Clinical Characteristics of Cognitive Impairment}

\begin{abstract}
To study the clinical characteristics of cognitive impairment in patients with hemorrhagic stroke, Birmingham cognitive screen test, mini-mental state examination, Montreal cognitive assessment was used to evaluate the cognitive function of acute hemorrhagic stroke and the incidence and clinical characteristics of cognitive impairment was evaluated. Seventy acute hemorrhagic stroke patients were enrolled in the study and the abnormal rates of Birmingham cognitive screen test, mini-mental state examination and Montreal cognitive assessment were 81.42, 33.34 and $41.5 \%$, respectively. Birmingham cognitive screen test showed that patients with acute hypertensive intracerebral hemorrhage have cognitive impairment in many aspects, especially in language function, memory, attention, executive function, number and application ability and the damage rate was more than $30 \%$. Different degrees of cognitive impairment involving multiple cognitive domains could occur in the acute stage of hemorrhagic stroke, among which language ability, memory, execution and application ability were the most common. Compared to minimental state examination and Montreal cognitive assessment, Birmingham cognitive screen test has a higher detection rate of cognitive impairment after hypertensive hemorrhage and a more comprehensive evaluation of cognitive function.
\end{abstract}

Key words: Stroke, cerebral infarction, hemorrhage, intracerebral

Vascular cognitive impairment (VCI) was a syndrome of mild cognitive decline or dementia caused by cerebrovascular disease (cerebral infarction or cerebral hemorrhage) or its related risk factors, which often cause one or more cognitive impairments such as memory, calculation, temporal and spatial orientation, executive ability ${ }^{[1]}$. Previous studies on VCI have mostly focused on ischemic stroke, but there were relatively few studies on cognitive impairment caused by intracerebral hemorrhage.

The neuropsychological scale was often used to evaluate the cognitive function of patients. However, most of the previous scales were aimed at Alzheimer's disease and there were deficiencies in the evaluation of VCI. The mini mental state examination (MMSE) $^{[2]}$ and the Montreal cognitive assessment test (MoCA $)^{[3]}$ are probably the most commonly used cognitive screening tests in the People's Republic of China, if not worldwide. However MMSE lacks sensitivity to detect patients with mild symptoms, especially those with visuospatial and executive function impairments ${ }^{[4]}$. The MoCA, though more sensitive than the $\mathrm{MMSE}^{[5]}$, remains limited. For example, being developed for dementia, the MoCA is not tuned to detect some of the common cognitive problems after stroke-notably visuospatial neglect and $\operatorname{apraxia}^{[6-9]}$.

Moreover, both the MMSE and the MoCA are highly verbal screens, with the majority of tests requiring good verbal abilities. This means that patients with aphasia found after stroke can fail the tests for reasons to do with language impairments and not the putative factors being tested (e.g. memory or attention). There is also a lack of specificity in assigning a problem to a specific cognitive function. These screens typically return an overall score, which cannot be easily used to direct treatment, as treatment needs to target the specific cognitive problem (e.g. therapy for spatial neglect differs from that for apraxia). 
The Birmingham cognitive screen $(\mathrm{BCoS})$ battery ${ }^{[10]}$ is a new instrument that was developed to screen individuals for cognitive problems after a stroke and to generate a cognitive profile across a range of cognitive domains such as attention and executive function, language, memory, number skills and praxis. Importantly, the test is designed to maximize inclusion of stroke patients, while generating test results that are uncontaminated by the co-occurrence of language or spatial attention problems, which can otherwise have a contaminating impact on performance. The reliability and validity of the Chinese version of the $\mathrm{BCoS}$ has been verified in Guangzhou ischemic stroke population ${ }^{[9]}$. In this study, $\mathrm{BCoS}$ was used to evaluate the cognitive impairment associated with hemorrhagic stroke.

\section{MATERIALS AND METHODS}

\section{Clinical subjects:}

All the patients included in this study were diagnosed with acute hypertensive intracerebral hemorrhage who were hospitalized in the Department of Neurology and Neurosurgery of Guangzhou First People's Hospital from July 2015 to April 2017. The inclusion criteria were, patients with age $\geq 50 \mathrm{y}$, those patients whose mother tongue is Cantonese or who are proficient in Cantonese, those with diagnosis of hypertensive intracerebral hemorrhage met the criteria set out in the 2015 guidelines for the diagnosis and treatment of intracerebral hemorrhage, those patients who can maintain attention for at least $45 \mathrm{~min}$, those patients who read the subject's instructions and signed a written informed consent form and those patients who are dextromanual.

The exclusion criteria included, those patients with central nervous system diseases such as stroke, epilepsy and Parkinson's disease, and patients previously diagnosed with dementia, normal intracranial pressure hydrocephalus, depression, organic mental disorders, mental disorders caused by substance dependence and schizophrenia. Patients with chronic cardiac insufficiency, thyroid hypothyroidism, moderate and severe anemia, and other medical diseases affecting cognitive function, systemic malignant tumors or intracranial benign tumors, other serious somatic diseases that affect the evaluation of cognitive function were excluded. Patients with unstable vital signs, those with attention span less than $45 \mathrm{~min}$ and patients or their families who did not give informed consent or refuse to sign the informed consent.
The clinical data of all patients were collected, including admission number, NIHSS, sex, date of birth, years of education, address, telephone, residence status, anamnesis, family history, personal history. All patients were assessed on cognitive function assessment scale within $14 \mathrm{~d}$ of onset followed by a trained neurologist, according to the order of BCoS scale, MMSE scale and MoCA scale. The language of $\mathrm{BCoS}$ was Cantonese, and its reliability and validity was verified in Guangzhou ischemic stroke population. The Chinese version of the MMSE scale was completed within $15 \mathrm{~min}$, with 23 points as the cut-off value, divided according to the number of years of education, including illiteracy $\leq 17$ points, primary school level $\leq 20$ points, secondary school level (including technical secondary school) $\leq 22$ points, college level (including junior college) $\leq 23$ points $^{[13]}$. MoCA adopts Hong Kong version ${ }^{[14]}$, each assessment was completed within 15 min, with a cut-off value of 23 , and the number of years of education if less than $12 \mathrm{y}$ plus 1 point.

\section{Statistical analysis:}

All patients' evaluation data were imported into an Excel database and the data was analyzed by SPSS 19.0. The mean \pm standard deviation was used in the measurement data which in accordance with normal distribution. The counting data was expressed by frequency or percentage, and the chi-square test was used for comparison between groups. For the comparison of qualitative data whose sample size is less than 40 , continuity correction was used. $\mathrm{P}<0.05$ indicates that the difference is significant, and all tests were bilateral tests.

\section{RESULTS AND DISCUSSION}

Finally, 70 patients were included in this study, including 50 males and 20 females. The average age was $66.31 \pm 11.31 \mathrm{y}$, the lowest age was $50 \mathrm{y}$ and the highest age was 91, and the average length of education was $8.10 \pm 3.66 \mathrm{y}$. All patients were of dextromanuality, including 21 with primary school education and below, 34 with middle school education and 15 with a university education or above. Among the 70 patients, 28 had damage in the left cerebral hemisphere, 35 in the right cerebral hemisphere, 5 in the cerebellum, 1 in the brainstem, and 1 in the bilateral cerebral hemispheres. All patients were proficient in Cantonese.

All patients completed the $\mathrm{BCoS}$ scale examination, 16 patients failed to complete MMSE, and 17 patients 
failed to complete the MOCA examination. Among the patients who had completed the $\mathrm{BCoS}$ scale, 6 patients could not complete MMSE or MoCA due to aphasia, and the remaining 10 patients could not complete MMSE or MoCA due to other reasons. The rates of cognitive impairment detected by MMSE, MoCA and $\mathrm{BCoS}$ were $33.34 \%(18 / 54), 41.5 \%(22 / 53)$ and $81.42 \%(57 / 70)$, respectively. Chi-square test was performed on the abnormality detection rates of MMSE and $\mathrm{BCoS}, \mathrm{MoCA}$ and $\mathrm{BCoS}$. It was found that the chi-square values of the two groups were all less than 0.05 , and the differences were statistically significant (Table 1). Of the 36 patients with normal MMSE scores, 25 patients were found to have at least one cognitive domain impairment by BCoS. Of the 31 patients with normal MoCA scores, 21 were found to have at least one cognitive domain impairment by $\mathrm{BCoS}$. In other words, more than $60 \%$ of the patients with normal MMSE or MoCA could find at least one cognitive domain impairment by $\mathrm{BCoS}$ examination, which mainly focused on attention, spatial neglect (apple deletion, tactile disappearance), language (sentence construction), memory (time and space, story recall and replay), controlled attention (auditory attention), and practical action ability (gesture).

According to the critical value of the Cantonese version of $\mathrm{BCoS}$ scale impairment of cognitive function in 70 patients with hypertensive intracerebral hemorrhage was counted. It was detected that there were many aspects of cognitive impairment in patients with acute hypertensive intracerebral hemorrhage, especially in language function, memory, attention, executive function, number, and application ability, and the impairment rate was more than $30 \%$. The damage including language subitems $(55.71 \%$ in sentence construction, $40.00 \%$ in sentence reading, $40.00 \%$ in character writing, $35.71 \%$ in non-word readingaccuracy), execution ability (47.14 \% in complex pattern copying, $41.42 \%$ in gesture recognition,

TABLE 1: COMPARISON OF ABNORMAL DETECTION RATE BETWEEN MMSE AND BCOS

\begin{tabular}{lcccc}
\hline & Normal cases & Abnormal cases & $\mathrm{X}^{2}$ & $\mathrm{P}$ value \\
\hline MMSE & 27 & 29 & 12.61 & 0.001 \\
$\mathrm{BCOS}$ & 13 & 57 & & \\
\hline
\end{tabular}

TABLE 2: COMPARISON OF ABNORMAL DETECTION RATE BETWEEN MOCA AND BCOS

\begin{tabular}{lcccc}
\hline & $\begin{array}{c}\text { Normal } \\
\text { cases }\end{array}$ & $\begin{array}{c}\text { Abnormal } \\
\text { cases }\end{array}$ & $\mathrm{x}^{2}$ & $\mathrm{P}$ value \\
\hline MoCA & 31 & 22 & & 0.000 \\
$\mathrm{BCOS}$ & 13 & 57 & 20.92 & \\
\hline
\end{tabular}

$35.71 \%$ in multi-step item use), attention and spatial neglect $(44.20 \%$ in apple deletion test, $41.40 \%$ in tactile evasion test, $40.00 \%$ in visual disappeared test), and controlled attention (44.28 \% in Birmingham rule conversion test), memory (52.77\% in task recall, $44.28 \%$ in time and space orientation), numbers (40.00\% in digital writing and $38.57 \%$ in digital reading). The specific impairments are shown in Table 2 .

The rate of language impairment in left hemisphere cerebral hemorrhage was higher, including attention and spatial neglect (apple deletion, visual disappearance, tactile disappearance), controlled attention (auditory attention), language (picture naming, sentence reading, nonword reading-accuracy, character writing), spatiotemporal orientation, digital writing, execution ability (complex graphic copying, gesture recognition). However, there was no difference between patients with left and right hemispheric lesions in several subitems, such as self-centered neglect, situational memory, digital reading, numerical calculation, and insight. The results were shown in Tables 3 and 4.

In this study, BCoS, MMSE, and MOCA were used to evaluate the cognitive function of patients with hypertensive intracerebral hemorrhage in the acute stage. The cognitive impairment detected by MMSE, MoCA, and BCoS were statistically significant. The detection rate of cognitive impairment in patients with hypertensive intracerebral hemorrhage by $\mathrm{BCoS}$ was significantly higher than that of the other two scales. Of the 36 patients with normal MMSE scores, 25 patients were found to have at least one cognitive domain impairment by $\mathrm{BCoS}$. Of the 31 patients with normal MoCA scores, 21 were found to have at least one cognitive domain impairment by $\mathrm{BCoS}$. The cognitive domain impairment mainly focused on attention, spatial neglect, language, memory, controlled attention, and practical action ability. This also showed that the clinical use of MMSE, MoCA scale for the cognitive screening of hemorrhagic stroke, patients' attention, spatial information reception ability and other aspects was easy to cause missed diagnosis. Among the patients who had completed the BCoS scale, 6 patients could not complete MMSE or MoCA due to aphasia. It is worth noting that both this study and previous studies had found that the patients with cerebral hemorrhage often have language dysfunction (the language subitem damage rate is $30-55 \%$ ). The evaluation of MMSE/ MoCA depended to a large extent on the integrity of patients' language functions. In case of patients with 


\begin{tabular}{|c|c|c|c|c|c|}
\hline \multirow[t]{2}{*}{ Age grouping } & \multicolumn{2}{|l|}{$50-69$ y } & \multicolumn{2}{|l|}{$\geq 70 y$} & \multirow[b]{2}{*}{$\begin{array}{c}\text { Total \% } \\
\text { impairment }\end{array}$} \\
\hline & $\begin{array}{c}\text { Functional } \\
\text { impairment cases/ } \\
\text { Total cases } \\
\end{array}$ & $\%$ & $\begin{array}{c}\text { Functional } \\
\text { impairment cases/ } \\
\text { Total cases } \\
\end{array}$ & $\%$ & \\
\hline \multicolumn{6}{|l|}{ Attention-spatial neglect } \\
\hline Apple cancellation (accuracy) & $24 / 45$ & $53.30 \%$ & $7 / 25$ & $28.00 \%$ & $44.20 \%$ \\
\hline Asymmetry (allocentric) (R, L) & $14 / 45$ & $31.10 \%$ & $2 / 25$ & $8.00 \%$ & $22.80 \%$ \\
\hline Asymmetry (egocentric) (R, L) & $0 / 45$ & $0.00 \%$ & $0 / 25$ & $0.00 \%$ & $0.00 \%$ \\
\hline Left visual unilateral & $17 / 45$ & $37.80 \%$ & $11 / 25$ & $44.00 \%$ & $40.00 \%$ \\
\hline Right visual unilateral & $10 / 45$ & $22.20 \%$ & $6 / 25$ & $24.00 \%$ & $22.80 \%$ \\
\hline Left visual bilateral & $15 / 45$ & $33.30 \%$ & $11 / 25$ & $44.00 \%$ & $37.10 \%$ \\
\hline Right visual bilateral & $14 / 45$ & $31.10 \%$ & $10 / 25$ & $40.00 \%$ & $34.20 \%$ \\
\hline Left tactile unilateral & $15 / 45$ & $33.30 \%$ & $1 / 25$ & $4.00 \%$ & $22.80 \%$ \\
\hline Right tactile unilateral & $12 / 45$ & $26.70 \%$ & $3 / 25$ & $12.00 \%$ & $21.40 \%$ \\
\hline Left tactile bilateral & $20 / 45$ & $44.40 \%$ & $9 / 25$ & $36.00 \%$ & $41.40 \%$ \\
\hline Right tactile bilateral & $15 / 45$ & $33.30 \%$ & $8 / 25$ & $32.00 \%$ & $32.85 \%$ \\
\hline \multicolumn{6}{|l|}{ Attention-controlled } \\
\hline Auditory attention accuracy & $18 / 45$ & $40.00 \%$ & $5 / 25$ & $20.00 \%$ & $32.85 \%$ \\
\hline Sustained attention index & $1 / 45$ & $2.00 \%$ & $4 / 25$ & $16.00 \%$ & $7.14 \%$ \\
\hline Auditory attention practice & $1 / 45$ & $2.00 \%$ & $0 / 25$ & $0.00 \%$ & $1.42 \%$ \\
\hline Auditory attention word recall & $9 / 45$ & $20.00 \%$ & $6 / 25$ & $24.00 \%$ & $21.42 \%$ \\
\hline Rule finding and set shifting accuracy & $21 / 45$ & $46.70 \%$ & $10 / 25$ & $40.00 \%$ & $44.28 \%$ \\
\hline Rule finding-rules found & $0 / 45$ & $0.00 \%$ & $0 / 25$ & $0.00 \%$ & $0.00 \%$ \\
\hline \multicolumn{6}{|l|}{ Language-spoken } \\
\hline Instruction comprehension & $7 / 45$ & $15.50 \%$ & $1 / 25$ & $4.00 \%$ & $11.42 \%$ \\
\hline Picture naming & $13 / 45$ & $28.90 \%$ & $2 / 25$ & $8.00 \%$ & $21.42 \%$ \\
\hline Sentence construction & $24 / 45$ & $53.30 \%$ & $15 / 25$ & $60.00 \%$ & $55.71 \%$ \\
\hline \multicolumn{6}{|l|}{ Language-read and written } \\
\hline Sentence reading-accuracy & $18 / 45$ & $40.00 \%$ & $10 / 25$ & $40.00 \%$ & $40.00 \%$ \\
\hline Nonword reading-accuracy & $16 / 45$ & $35.30 \%$ & $9 / 25$ & $36.00 \%$ & $35.71 \%$ \\
\hline Character writing & $19 / 45$ & $42.20 \%$ & $9 / 25$ & $36.00 \%$ & $40.00 \%$ \\
\hline \multicolumn{6}{|l|}{ Memory-orientation } \\
\hline Personal & $6 / 45$ & $13.30 \%$ & $2 / 25$ & $8.00 \%$ & $11.42 \%$ \\
\hline Time and space & $24 / 45$ & $53.30 \%$ & $7 / 25$ & $28.00 \%$ & $44.28 \%$ \\
\hline \multicolumn{6}{|l|}{ Memory-episodic } \\
\hline Story free-recall 1 & $11 / 45$ & $24.40 \%$ & $7 / 25$ & $28.00 \%$ & $25.71 \%$ \\
\hline Story free-recognition 1 & $11 / 45$ & $24.40 \%$ & $9 / 25$ & $36.00 \%$ & $28.57 \%$ \\
\hline Story free-recall 2 & $10 / 45$ & $22.20 \%$ & $8 / 25$ & $32.00 \%$ & $25.71 \%$ \\
\hline Story free-recognition 2 & $12 / 45$ & $26.70 \%$ & $8 / 25$ & $32.00 \%$ & $28.57 \%$ \\
\hline Task-recall & $17 / 45$ & $37.80 \%$ & $21 / 25$ & $84.00 \%$ & $52.77 \%$ \\
\hline \multicolumn{6}{|l|}{ Number } \\
\hline Reading & $19 / 45$ & $42.20 \%$ & $8 / 25$ & $32.00 \%$ & $38.57 \%$ \\
\hline Writing & $19 / 45$ & $42.20 \%$ & $9 / 25$ & $36.00 \%$ & $40.00 \%$ \\
\hline Calculation & $7 / 45$ & $15.50 \%$ & $4 / 25$ & $16.00 \%$ & $15.71 \%$ \\
\hline \multicolumn{6}{|l|}{ Praxis-action } \\
\hline Figure copy & $23 / 45$ & $51.10 \%$ & $10 / 25$ & $40.00 \%$ & $47.14 \%$ \\
\hline Multistep object use & $16 / 45$ & $35.50 \%$ & $9 / 25$ & $36.00 \%$ & $35.71 \%$ \\
\hline Gesture production & $21 / 45$ & $46.70 \%$ & $2 / 25$ & $8.00 \%$ & $32.85 \%$ \\
\hline Gesture recognition & $17 / 45$ & $37.80 \%$ & $12 / 25$ & $48.00 \%$ & $41.42 \%$ \\
\hline Imitation & $15 / 45$ & $33.30 \%$ & $2 / 25$ & $8.00 \%$ & $24.28 \%$ \\
\hline
\end{tabular}

aphasia, there were obstacles in the reception and output of information, which often affected the judgment of other cognitive subitems such as MoCA or MMSE. The evaluation of the MMSE/MoCA mainly depended on the integrality of linguistic function. The $\mathrm{BCoS}$ could better solve the above problems. Most of the prompts of 


\begin{tabular}{|c|c|c|}
\hline$x^{2}$ test & & \\
\hline \multicolumn{3}{|l|}{ Attention-spatial neglect } \\
\hline Apple cancellation (accuracy) & 6.760 & 0.009 \\
\hline Asymmetry (allocentric) $(R, L)$ & 0.477 & 0.490 \\
\hline Asymmetry (egocentric) (R, L) & NA & NA \\
\hline Left visual unilateral & 2.363 & 0.136 \\
\hline Right visual unilateral & 0.039 & 0.843 \\
\hline Left visual bilateral & 4.062 & 0.044 \\
\hline Right visual bilateral & 0.877 & 0.349 \\
\hline Left tactile unilateral & 1.176 & 0.278 \\
\hline Right tactile unilateral & 6.998 & 0.012 \\
\hline Left tactile bilateral & 4.200 & 0.040 \\
\hline Right tactile bilateral & 2.057 & 0.151 \\
\hline \multicolumn{3}{|l|}{ Attention-controlled } \\
\hline Auditory attention accuracy & 3.889 & 0.049 \\
\hline Sustained attention index & 0.553 & 0.457 \\
\hline Auditory attention practice & 0.894 & 0.344 \\
\hline Auditory attention word recall & 0.556 & 0.456 \\
\hline Rule finding and set shifting accuracy & 0.630 & 0.427 \\
\hline \multicolumn{3}{|l|}{ Language-spoken } \\
\hline Instruction comprehension & 1.210 & 0.449 \\
\hline Picture naming & 10.705 & 0.001 \\
\hline Sentence construction & 1.197 & 0.274 \\
\hline \multicolumn{3}{|l|}{ Language-read and written } \\
\hline Sentence reading-accuracy & 4.062 & 0.044 \\
\hline Non-word reading-accuracy & 7.754 & 0.005 \\
\hline Character writing & 6.563 & 0.010 \\
\hline \multicolumn{3}{|l|}{ Memory-orientation } \\
\hline Personal & 3.465 & 0.063 \\
\hline Time and space & 16.313 & 0.000 \\
\hline \multicolumn{3}{|l|}{ Memory-episodic } \\
\hline Story free-recall 1 & 0.094 & 0.759 \\
\hline Story free-recognition 1 & 1.059 & 0.304 \\
\hline Story free-recall 2 & 0.695 & 0.404 \\
\hline Story free-recognition 2 & 1.403 & 0.236 \\
\hline Task-recall & 1.484 & 0.223 \\
\hline \multicolumn{3}{|l|}{ Number } \\
\hline Reading & 0.003 & 0.954 \\
\hline Writing & 7.862 & 0.005 \\
\hline Calculation & 0.149 & 0.700 \\
\hline \multicolumn{3}{|l|}{ Praxis-action } \\
\hline Figure copy & 4.585 & 0.032 \\
\hline Multistep object use & 1.484 & 0.223 \\
\hline Gesture production & 1.397 & 0.237 \\
\hline Gesture recognition & 8.036 & 0.005 \\
\hline Imitation & 2.832 & 0.092 \\
\hline
\end{tabular}

the $\mathrm{BCoS}$ were short and high-frequency words, which were equipped with a subject manual. All aphasia patients who were unable to answer questions freely can complete the corresponding content test by reading and choosing the corresponding text answers, so that they can avoid the influence of impaired language function as far as possible and truly reflect the status of other cognitive subitems of the patients.

In addition, compared with MMSE/MoCA, BCoS could comprehensively and specifically evaluate the impairment of each cognitive domain of the subjects, which was more conducive to the later rehabilitation treatment. National institute for health and clinical excellence guidelines for long-term rehabilitation after stroke indicate that for acute stroke patients, a comprehensive assessment including cognitive assessment should be completed as quickly as possible, including attention, memory, spatial ability, disability, perception ${ }^{[13]}$. The vascular cognitive impairment guidelines of China also recommend evaluating patients with vascular cognitive impairment, including memory, attention to executive function, visual space structure function. Therefore, an effective, comprehensive, and sensitive cognitive assessment scale is particularly important clinically. As a new and advanced assessment tool, the reliability and validity of $\mathrm{BCoS}$ had been verified in the UK and Guangdong, China. It has been proved to be a comprehensive and effective cognitive assessment tool ${ }^{[9]}$, which could provide a comprehensive cognitive examination for patients and provide a basis for clinical treatment and neurorehabilitation, thus it is worth further popularizing in clinical practice.

Using the Cantonese version of $\mathrm{BCoS}$, the impairment of cognitive function in 70 patients with hypertensive intracerebral hemorrhage was counted. It was observed that many aspects of cognitive impairment could occur in patients with acute hypertensive intracerebral hemorrhage, especially in language function, memory, attention, executive function, number, and application ability, and the rate of occurrence was more than $30 \%$. And the damage including language subitems, execution ability, attention and spatial neglect and controlled attention, memory and numbers. It is consistent with the results of previous studies on cognitive screening of hemorrhagic stroke by scholars at home and abroad ${ }^{[16,17]}$. However, the conclusions of most previous studies were not uniform and BCoS was used to comprehensively evaluate hemorrhagic stroke in this study, which ovecame the shortcomings of previous studies.

The present study also found that there was no difference between the left and right hemispheres of episodic memory, spatial neglect, digital reading and computing. Consistent with previous reports ${ }^{[12]}$, it was 
observed that lesions in the left cerebral hemisphere were more prone to functional impairment of multiple cognitive domains, including language, attention, and executive function, such as picture naming, nonword reading-accuracy, character writing. Episodic memory was the memory of events (situations) experienced by individuals at a certain time and place on the basis of time and space. In the past, it was thought that memory was mainly related to the dominant hemispheres. Broca's area, in the frontal lobe of the left hemisphere is found to be largely affected in stroke patients and so is the speech production. However, recent studies have also supported the importance of non-dominant hemispheres in the maintenance of episodic memory function. For example, a PET study ${ }^{[18]}$ conducted in 2009 found that episodic memory was different from other types of memory. Its neural structure was not only based on the medial nucleus of the temporal lobe, but also related to multiple brain regions of both hemispheres. The complete functional network between the bilateral hemispheres was involved in the formation of episodic memory. This study also supported this view from the study of lesions-function matching.

This study showed that there was no difference in the abnormal rate of egocentric neglect in both hemispheres. Egocentric neglect refers to self-centered neglect of spatial information on one side ${ }^{[19,20]}$. It was a common cognitive impairment, after brain injury such as stroke. Foreign studies have shown that the incidence in stroke patients can reach $25-50 \%{ }^{[21]}$, and about $1 / 3$ of acute neglect patients can be converted to chronic neglect ${ }^{[22]}$. Many studies have reported that patients with damage to the right cerebral hemisphere have a higher incidence and severity of neglect, but some studies have shown that there was no difference in the incidence of spatial neglect between patients with damage to the left and right hemispheres ${ }^{[21,23]}$. However, there was a relative lack of research in this area in our country. The present results also supported the possibility of egocentric neglect in the right hemisphere damage. Studies abroad used Ota's discriminative cancellation task combined with line bisection task to conduct DWI studies in patients with acute stroke and TIA. They found that there was no difference in the incidence of neglect between patients with left and right cerebral hemispheres ${ }^{[21]}$. It was speculated that this might be due to the fact that the ability to receive visual spatial information depended on the functional connection between the bilateral hemispheres, not just on the functional integrity of the brain near the parietal lobe of the right hemisphere. On the other hand, the hemispheric damage in patients with hypertensive intracerebral hemorrhage might be mainly subcortical structural damage, but less related to the involvement of right angular gyrus and supramarginal gyrus which were closely related to neglect. Next, the subgroup analysis of specific lesions will help to clarify this problem.

Therefore, it was detected that the main neural structural basis of cognitive abilities such as language, application, execution, and numbers were located in the left hemisphere, while the integrity of cognitive functions such as episodic memory, self-centered neglect, digital reading, and computing may depend more on the integrity of functional connections between bilateral hemispheres. In conclusion, different degrees of cognitive impairment involving multiple cognitive domains could occur in the acute stage of hypertensive intracerebral hemorrhage, among which language ability, memory, executive ability, and application ability was the commonest. Compared with MMSE and MoCA, BCoS has a higher detection rate of cognitive impairment after hypertensive hemorrhage and a more comprehensive evaluation of cognitive function. The dominant hemispheres was more prone to multiple cognitive domain impairment, including language, but episodic memory, self-centered neglect, digital reading and computing functions were all related to bilateral cerebral hemispheres. This may be achieved through the functional connection between different parts of the bilateral hemispheres, and there was a certain degree of compensatory mechanism between different functional parts, which needs to be further confirmed by expanding the sample size.

\section{Acknowledgements:}

This study was supported by Guangzhou Planned Project of Science and Technology (No. 2016201604030018) and Innovative Clinical Technique of Guangzhou (2019GX06).

\section{Conflict of interests:}

The authors declared no conflict of interest.

\section{REFERENCES}

1. Hachinski V, Iadecola C, Petersen RC, Breteler MM, Nyenhuis DL, Black, SE, et al. National Institute of Neurological Disorders and Stroke-Canadian Stroke Network vascular cognitive impairment harmonization standards. Stroke 2006;37(9):2220-41.

2. Folstein MF, Folstein SE, McHugh PR. "Mini-mental state.” J Psychiatr Res 1975;12:189-98.

3. Nasreddine ZS, Phillips NA, Bédirian V. The Montreal Cognitive Assessment, MoCA: A brief screening tool for mild 
cognitive impairment. J Am Geriatr Soc 2005;53:695-9.

4. Dong Y, Sharma VK, Chan BPL. The Montreal Cognitive Assessment (MoCA) is superior to the Mini-Mental State Examination (MMSE) for the detection of vascular cognitive impairment after acute stroke. J Neurol Sci 2010;299:15-8.

5. Pendlebury ST, Cuthbertson FC, Welch SJV, Mehta Z, Rothwell PM. Underestimation of Cognitive Impairment by Mini-Mental State Examination versus the Montreal Cognitive Assessment in Patients with Transient Ischemic Attack and Stroke: A Population-Based Study. Stroke 2010;41(6):1290-3.

6. Bickerton W-L, Riddoch MJ, Samson D, Balani AB, Mistry B, Humphreys GW. Systematic assessment of apraxia and functional predictions from the Birmingham Cognitive Screen. J Neurol Neurosurg Psychiatry. 2012;83(5):513-21.

7. Chechlacz M, Rotshtein P, Bickerton W-L, Hansen PC, Deb S, Humphreys GW. Separating neural correlates of allocentric and egocentric neglect: distinct cortical sites and common white matter disconnections. Cogn Neuropsychol 2010;27(3):277303.

8. Chen H, Pan X, Lau JKL. Lesion-symptom mapping of a complex figure copy task: A large-scale PCA study of the BCoS trial. NeuroImage Clin. 2016;11:622-34.

9. Pan X, Chen H, Bickerton WL. Preliminary findings on the reliability and validity of the Cantonese Birmingham cognitive screen in patients with acute ischemic stroke. Neuropsychiatr Dis Treat 2015;11:2377-90.

10. Titova E, Ostrowski RP, Zhang JH, Tang J. Experimental models of subarachnoid hemorrhage for studies of cerebral vasospasm. Neurol Res 2009;31:568-81.

11. Morris PG, Wilson JTL, Dunn LT, Nicoll JAR. Apolipoprotein E polymorphism and neuropsychological outcome following subarachnoid haemorrhage. Acta Neurol Scand 2004;109:2059.

12. Bickerton W-L, Demeyere N, Francis D. The BCoS Cognitive Profile Screen: Utility and predictive value for stroke. Neuropsychology 2015;29(4):638-48

13. Roselli F, Tartaglione B, Federico F, Lepore V, Defazio G, Livrea P. Rate of MMSE score change in Alzheimer's disease: Influence of education and vascular risk factors. Clin Neurol Neurosurg 2009;111(4):327-30

14. Yeung PY, Wong LL, Chan CC, Leung JLM, Yung CY. A validation study of the Hong Kong version of Montreal Cognitive Assessment (HK-MoCA) in Chinese older adults in Hong Kong. Hong Kong Med J 2014;20(6):504-10.
15. Drummond A, Wade DT. National Institute for Health and Care Excellence stroke rehabilitation guidance - Is it useful, usable, and based on best evidence? Clin Rehabil 2014;28(6):523-9.

16. Kim JH, Silberfein CM, Westerveld M, Buchanan CP, Spencer DD. Right Hemisphere Mediation of Verbal Learning and Memory in Acquired Right Hemisphere Speech Dominant Patients. J Int Neuropsychol Soc 1995;1(6):554-60.

17. Bugiani O, Conforto C, Sacco G. Aphasia in thalamic haemorrhage. Lancet 1969;1(7604):1052.

18. Ozeki T, Azuma F, Makiura Y, Nokura K, Yamamoto H, Kamiya M. Pure agraphia with topographical disorientation caused by right hemisphere lesion. Clin Neurol 2008;48(7):505-8.

19. Demeyere N, Gillebert C, Loftus L, Humphreys G. Egocentric and allocentric neglect after right and left hemisphere lesions in a large scale neglect study of acute stroke patients: Prevalence and recovery. J Vis 2015;15(12):179.

20. Bickerton WL, Samson D, Williamson J, Humphreys GW. Separating forms of neglect using the Apples Test: Validation and functional prediction in chronic and acute stroke. Neuropsychology 2011;25:567-80.

21. Kamtchum Tatuene J, Allali G, Saj A. Incidence, Risk Factors and Anatomy of Peripersonal Visuospatial Neglect in Acute Stroke. Eur Neurol 2016;75(3-4):157-63.

22. Karnath HO, Rennig J, Johannsen L, Rorden C. The anatomy underlying acute versus chronic spatial neglect: A longitudinal study. Brain 2011;134(3):903-12.

23. Beis J-M, Keller C, Morin N. Right spatial neglect after left hemisphere stroke: qualitative and quantitative study. Neurology 2004;63(9):1600-5.

24. Siler DA. Soluble Epoxide Hydrolase in Hydrocephalus, Cerebral Edema, and Vascular Inflammation After Subarachnoid Hemorrhage. Stroke 2015;46:1916-22.

25. Li M. Methazolamide improves neurological behavior by inhibition of neuron apoptosis in subarachnoid hemorrhage mice. Sci Rep 2016;6:35055.

This is an open access article distributed under the terms of the Creative Commons Attribution-NonCommercial-ShareAlike 3.0 License, which allows others to remix, tweak, and build upon the work non-commercially, as long as the author is credited and the new creations are licensed under the identical terms

This article was originally published in a special
issue, "Biomedical Research in Healthcare Setting"
Indian J Pharm Sci 2020:82(3)Spl issue5;130-136

